\title{
Stochastic Modeling of High-Speed Data Links with Nonlinear Dynamic Terminations
}

\author{
Paolo Manfredi*, Igor S. Stievano*, Dries Vande Ginste ${ }^{\dagger}$, Daniël De Zutter ${ }^{\dagger}$, and Flavio G. Canavero* \\ *EMC Group, Department of Electronics and Telecommunications, Politecnico di Torino \\ Corso Duca degli Abruzzi 24, 10129 Torino, Italy \\ E-mail: paolo.manfredi@polito.it \\ ${ }^{\dagger}$ Electromagnetics Group, Department of Information Technology, Ghent University \\ Sint-Pietersnieuwstraat 41, 9000 Gent, Belgium
}

\begin{abstract}
This paper addresses the statistical modeling and simulation of high-speed interconnects with uncertain physical properties and nonlinear dynamical terminations. The proposed approach is based on the expansion of voltage and current variables in terms of orthogonal polynomials of random variables. It extends the available literature results on the generation of an augmented deterministic SPICE equivalent of the stochastic link to the case in which the terminations are nonlinear and dynamical, like those modeling IC buffers. A single and standard SPICE simulation of the aforementioned equivalent circuit allows to efficiently compute the expansion coefficients that provide statistical information pertinent to the interconnect response. The feasibility and strength of the approach are demonstrated by means of a coupled microstrip interconnect with drivers and receivers.
\end{abstract}

Index Terms-Circuit simulation, nonlinear, polynomial chaos, signal integrity, SPICE, statistical analysis, transmission lines.

\section{INTRODUCTION}

Nowadays, the trend of increasing the operating frequency while shrinking the physical dimensions of digital communication links unavoidably demands for the inclusion of uncertainties in the interconnect properties when assessing the signal integrity and electromagnetic compatibility. In this framework, the availability of methods and tools for the stochastic simulation of circuits and interconnects becomes a key design resource. A standard solution provided by commercial (e.g. SPICE-type) simulators to tackle this problem are the widelyused Monte Carlo (MC)-like approaches. The above class of methods, however, requires a large number of simulation runs, with a detrimental effect on the simulation time.

Recent literature proposes an alternative set of clever solutions [1]-[5], providing improved efficiency based on the socalled polynomial chaos (PC) approach [6]. According to PC, the voltages and currents of a circuit, which become random variables due to the uncertainty affecting the circuit elements, are expanded into a series of orthonormal polynomials. The above interpretation facilitates the computation of accurate statistical information via the determination of the unknown expansion coefficients. For a general overview, the readers are referred to [1], where the proposed PC-based method has been applied for the first time to the frequency-domain stochastic solution of on-board interconnects. Further enhancements mainly focused on the generation of SPICE-compatible equivalent circuits for time-domain analysis [2] and/or on the inclusion of static nonlinear elements [3]-[5].

This paper extends the above results to the time-domain stochastic analysis of high-speed interconnects with nonlinear dynamical terminations. The proposed methodology allows to include the numerical models of digital integrated circuits (ICs) in the statistical assessment of data links affected by random parameter variability. In the advocated approach, the devices are considered as multiport black-boxes, thus allowing to include any available (e.g., transistor-level or behavioral) description of digital ICs. To further speed-up the simulation, accurate and efficient state-of-the-art IC macromodels [7] are therefore used instead of transistor-level descriptions.

\section{The Polynomial Chaos Approach}

Fig. 1 illustrates a typical high-speed transmission link consisting of two coupled interconnects, each with a driver and a receiver. The interconnect section is modeled as a coupled transmission line of length $\mathcal{L}$, with pertinent $2 \times 2$ per-unitlength (p.u.l.) resistance $\mathbf{R}$, inductance $\mathbf{L}$, conductance $\mathbf{G}$ and capacitance $\mathbf{C}$ matrices. The drivers and receivers may be described by either a transistor-level circuit or equivalent (behavioral) models [7].

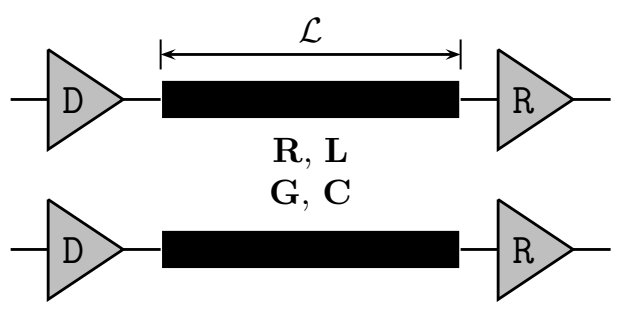

Fig. 1. Pictorial illustration of a high-speed transmission link with drivers and receivers.

Suppose that the link is affected by $d$ random parameters, collected into $\mathbf{x}=\left[x_{1}, \ldots, x_{d}\right]^{T}$, which for the sake of simplicity we assume to be normally distributed. We first express the vector $\mathbf{x}$ in terms of standard normal variables $\boldsymbol{\xi}=\left[\xi_{1}, \ldots, \xi_{d}\right]^{T}:$

$$
\mathbf{x}=\boldsymbol{\mu}+\boldsymbol{\Sigma} \boldsymbol{\xi}
$$


where $\boldsymbol{\mu}=\left[\mu_{1}, \ldots, \mu_{d}\right]^{T}$ (with $\mu_{i}$ the average value of $x_{i}$ ) and $\boldsymbol{\Sigma}=\operatorname{diag}\left(\left[\sigma_{1}, \ldots, \sigma_{d}\right]\right)$ (with $\sigma_{i}$ the corresponding standard deviation). Should the random parameters $\mathrm{x}$ be correlated, $\boldsymbol{\Sigma}$ becomes a full matrix obtained via the eigenvalue decomposition of the correlation matrix. Generalization to arbitrary probability distributions is available e.g. in [4].

\section{A. The PC Expansion}

The variability of the link properties renders the circuit voltages and currents $\boldsymbol{\xi}$-dependent, i.e. stochastic. The traditional approach to collect statistical information in commercial circuit solvers (e.g. [8]) is to simulate the link for many random configurations. This approach is known as the MC method and is computationally inefficient. An alternative strategy was proposed based on PC, where voltages and currents are expanded as [4]

$$
v_{n}(t, \boldsymbol{\xi}) \approx \sum_{k=0}^{P} v_{n k}(t) \varphi_{k}(\boldsymbol{\xi}), i_{n}(t, \boldsymbol{\xi}) \approx \sum_{k=0}^{P} i_{n k}(t) \varphi_{k}(\boldsymbol{\xi}),
$$

with the subscript $n$ denoting the node/branch. The coefficients provide direct statistical information [6]. The functions $\left\{\varphi_{k}\right\}$ form a complete basis of orthonormal polynomials w.r.t. the inner product

$$
\langle f, g\rangle=\int_{\mathbb{R}^{d}} f(\boldsymbol{\xi}) g(\boldsymbol{\xi}) w(\boldsymbol{\xi}) d \boldsymbol{\xi},
$$

with $w(\boldsymbol{\xi})=\mathrm{e}^{-\frac{1}{2} \boldsymbol{\xi}^{T} \boldsymbol{\xi}}$ the joint probability density function (PDF) of the random variables $\boldsymbol{\xi}$.

In order to calculate the unknown PC coefficients in (2), pertinent deterministic equations are obtained via a stochastic Galerkin method (SGM) [6], and then given a circuit interpretation that can be simulated via standard SPICE-type software [4]. This single deterministic simulation usually turns out to substantially outperform the MC analysis.

\section{B. Modeling of Stochastic Transmission-Line Elements}

The modeling of stochastic transmission-line elements was addressed in [2] and is summarized in this section. The PC expansions (2) of the line voltages and currents are substituted into the governing equations (i.e., the Telegrapher's equations [9]). Then, the resulting equations are tested using the same polynomial basis $\left\{\varphi_{m}\right\}_{m=0}^{P}$ and weighed using the inner product (3). This procedure transforms the original stochastic equations into a $(P+1)$-augmented set of deterministic equations in the unknown coefficients:

$$
\begin{aligned}
\frac{d}{d z} \hat{\tilde{\mathbf{V}}}(z, \omega) & =-[\widetilde{\mathbf{R}}(\omega)+j \omega \widetilde{\mathbf{L}}(\omega)] \hat{\tilde{\mathbf{I}}}(z, \omega), \\
\frac{d}{d z} \hat{\tilde{\mathbf{I}}}(z, \omega) & =-[\widetilde{\mathbf{G}}(\omega)+j \omega \widetilde{\mathbf{C}}(\omega)] \hat{\tilde{\mathbf{V}}}(z, \omega),
\end{aligned}
$$

with $\hat{\tilde{\mathbf{V}}}$ and $\hat{\widetilde{\mathbf{I}}}$ collecting the phasors of the line voltage and current PC coefficients, respectively. The new $(P+1)$ augmented p.u.l. matrices $\widetilde{\mathbf{R}}, \widetilde{\mathbf{L}}, \widetilde{\mathbf{G}}$, and $\widetilde{\mathbf{C}}$ are readily calculated based on the statistics of the link properties [2]. The equations (4) describe a (provably passive) transmissionline section of increased size, that can still be simulated in a standard circuit solver (typically, via convolution-based techniques [8]). However, pertinent and consistent equations must be derived for the components connected to the new line terminations.

\section{Modeling of Nonlinear Dynamic Multi-Terminal Elements}

So far, only linear terminations [2] or two- and threeterminal nonlinear elements with static characteristic (e.g., diodes and transistors) were modeled [4]. In this contribution, a far more general framework is provided, where the nonlinear component has an arbitrary number of terminals and is not given as a single element, but rather described in terms of a subcircuit.

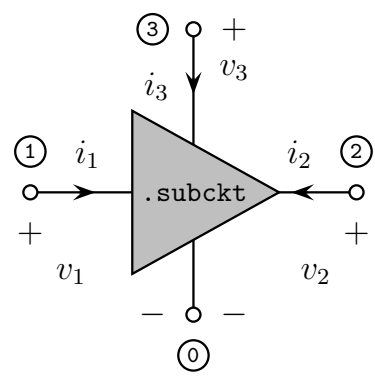

(a)

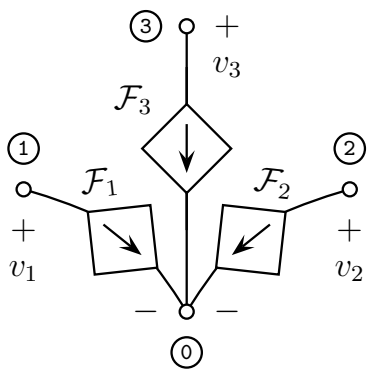

(b)
Fig. 2. Driver model with terminals definition (a) and equivalent representation (b).

For our discussion, we focus on the driver in Fig. 2(a), having four terminals, one of which (0) is the reference. The internal description of the driver is irrelevant for our purpose. Without loss of generality, we assume that the model is fully voltage-controlled and we express the currents entering terminals 1,2 and 3 as

$$
\begin{aligned}
& i_{1}=\mathcal{F}_{1}\left(v_{1}, v_{2}, v_{3}, t\right), \\
& i_{2}=\mathcal{F}_{2}\left(v_{1}, v_{2}, v_{3}, t\right), \\
& i_{3}=\mathcal{F}_{3}\left(v_{1}, v_{2}, v_{3}, t\right),
\end{aligned}
$$

where $\mathcal{F}_{1,2,3}$ represent the current-to-voltage responses of the driver. The above equations (5) can be represented via voltagedependent current sources, as illustrated in Fig. 2(b). This approach is readily generalized to an arbitrary number of terminals.

Replacing the PC expansions (2) of the voltages and currents into (5) produces

$$
\sum_{k=0}^{P} i_{n k}(t) \varphi_{k}(\boldsymbol{\xi})=\mathcal{F}_{n}\left(\hat{v}_{1}(t, \boldsymbol{\xi}), \hat{v}_{2}(t, \boldsymbol{\xi}), \hat{v}_{3}(t, \boldsymbol{\xi})\right),
$$

$(n=1,2,3)$ with

$$
\hat{v}_{n}(t, \boldsymbol{\xi})=\sum_{k=0}^{P} v_{n k}(t) \varphi_{k}(\boldsymbol{\xi}) .
$$

Application of the SGM allows to find a deterministic equation for the $m$ th current coefficient (with $m=0, \ldots, P$ ):

$$
i_{n m}(t)=\int_{\mathbb{R}^{d}} \mathcal{F}_{n}\left(\hat{v}_{1}(t, \boldsymbol{\xi}), \hat{v}_{2}(t, \boldsymbol{\xi}), \hat{v}_{3}(t, \boldsymbol{\xi})\right) \varphi_{m}(\boldsymbol{\xi}) d \boldsymbol{\xi} .
$$


It should be noted that (8) is deterministic because the variable $\boldsymbol{\xi}$ in the right-hand side is integrated out. However, as (8) is an integral equation, it cannot be implemented in a circuit solver in its present form. Hence, the next step is the discretization of the integral in (8) through a quadrature rule [3], e.g., with a set of $Q$ quadrature points $\left\{\boldsymbol{\xi}_{q}\right\}$ and corresponding weights $w_{q}$, yielding

$$
i_{n m}(t)=\sum_{q=1}^{Q} \mathcal{F}_{n}\left(\hat{v}_{1 q}(t), \hat{v}_{2 q}(t), \hat{v}_{3 q}(t)\right) a_{m q} w_{q},
$$

where we defined $a_{m q}=\varphi_{m}\left(\boldsymbol{\xi}_{q}\right)$ and

$$
\hat{v}_{n q}(t)=\hat{v}_{n}\left(t, \boldsymbol{\xi}_{q}\right)=\sum_{k=0}^{P} v_{n k}(t) \varphi_{k}\left(\boldsymbol{\xi}_{q}\right)=\sum_{k=0}^{P} v_{n k}(t) a_{k q} .
$$

The above equation (9) is rewritten as

$$
i_{n m}(t)=\sum_{q=1}^{Q} j_{n q}(t) b_{m q},
$$

where $b_{m q}=a_{m q} w_{q}$, whilst the nonlinear current

$$
j_{n q}(t)=\mathcal{F}_{n}\left(\hat{v}_{1 q}(t), \hat{v}_{2 q}(t), \hat{v}_{3 q}(t)\right)
$$

is the current flowing in the $n$th driver terminal when voltages $\hat{v}_{1 q}(t), \hat{v}_{2 q}(t)$ and $\hat{v}_{3 q}(t)$ are applied to its terminals.

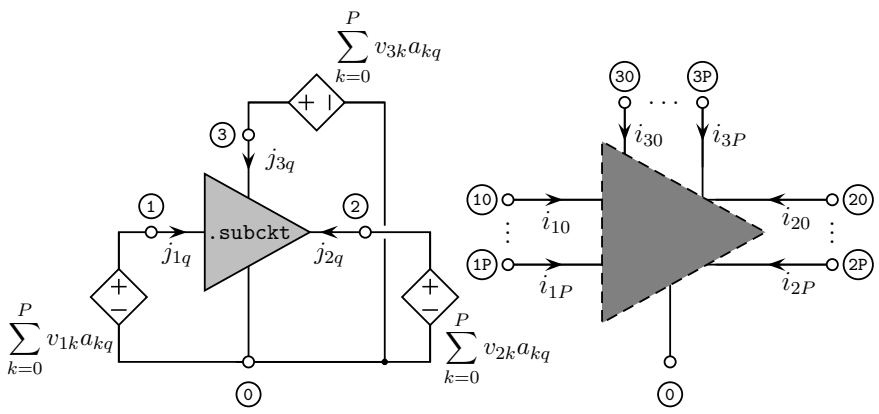

(a)

(b)

Fig. 3. Driver model for the deterministic augmented circuit. Circuit for the calculation of the nonlinear currents $j_{n q}$ (a) and overall representation (b).

Recalling the definition (10), the currents $j_{n q}$ are obtained by means of the circuit in Fig. 3(a), where voltages $v_{n k}$, with $n=1,2,3$ and $k=0, \ldots, P$, are the terminal voltages appearing in Fig. 3(b). It should be noted that $Q$ of these circuits (for $q=1, \ldots, Q)$ are necessary, and therefore the deterministic model consists of $Q$ replicas of the driver model. Finally, the terminal currents $i_{n m}$ in Fig. 3(b) are obtained through the linear combination (11) by means of dependent current sources, similar to Fig. 2(b). Expressions (11) represents the sought-for terminal equations for the augmented transmission line in (4), and are implemented in SPICE-type simulators via standard components. The $m$ th line termination is connected to the pertinent $m$ th terminal in Fig. 3(b).

\section{Application EXAMPLE}

To validate the proposed methodology, the link in Fig. 1 is considered. The interconnect is a coupled microstrip line with a trace width of $100 \mu \mathrm{m}$, a trace thickness of $35 \mu \mathrm{m}$, a trace separation of $80 \mu \mathrm{m}$, and a substrate thickness of $500 \mu \mathrm{m}$. Due to process variations, the trace separation and the substrate thickness are assumed to be two Gaussian random variables with a relative standard deviation of $10 \%$ each. The two drivers in the link represent the I/O transceivers of a $512-\mathrm{Mb}$ Flash memory chip and are modeled by means of equivalent and computationally-efficient SPICE-compatible behavioral macromodels [10]. One driver is active and produces a pulse with a risetime of $1 \mathrm{~ns}$, whilst the other driver is quiet and kept to the "low" state. The receivers are modeled with two shunt capacitances of $5 \mathrm{pF}$. Not shown in Fig. 1 is the RL power supply network for the drivers $\left(V_{D D}=1.8 \mathrm{~V}\right)$, with $R=10 \mathrm{~m} \Omega$ and $L=2 \mathrm{nH}$.

The simulations are carried out using HSPICE [8] on an ASUS U30S laptop with an Intel(R) Core(TM) i3-2330M and $\mathrm{CPU}$ running at $2.20 \mathrm{GHz}$. Reference results are generated by means of a MC analysis, using the available HSPICE feature and considering 1000 samples.

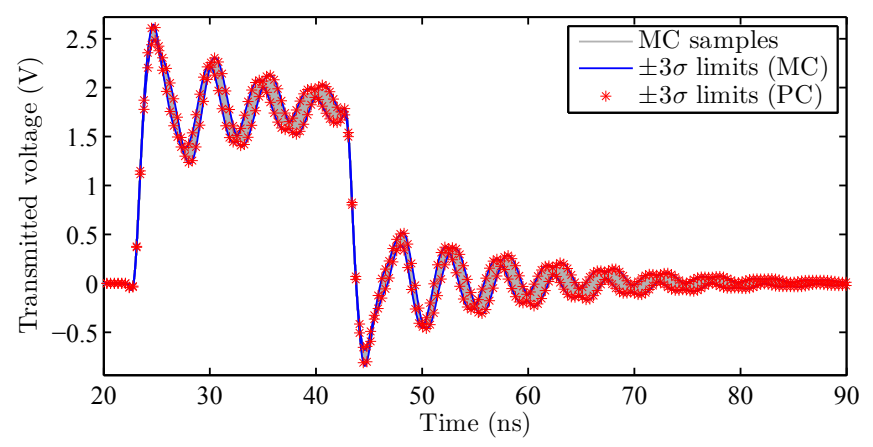

Fig. 4. Voltage transmitted to the far-end termination of the link in Fig. 1. Gray area: random fluctuation of the link response; blue lines and red markers: $\pm 3 \sigma$ bounds computed with MC and PC, respectively.

Fig. 4 shows the voltage transmitted to the far-end termination of the active line. The gray area results from the superposition of a subset of $100 \mathrm{MC}$ samples and provides a qualitative idea of the fluctuation of the link response due to the variability. The blue lines and the red markers provide a more quantitative information, namely the $\pm 3 \sigma$ bounds (with $\sigma$ the standard deviation), obtained by means of MC and PC, respectively. Excellent agreement can be appreciated. For the PC-based simulation, which took $141 \mathrm{~s}, P=5$ and $Q=9$ were considered. The MC analysis required 12883 s. Hence, an impressive speed-up factor of about $90 \times$ is achieved.

From the PC expansions, statistical functions like PDFs can be extracted as well. Fig. 5 compares the PDF of the maximum overshoot at the far-end termination (occurring at $t=24.7 \mathrm{~ns}$ ) obtained from the MC samples (gray bars) against the one estimated from the PC expansion (red line). The resolution of the MC result is poor due to the limited 


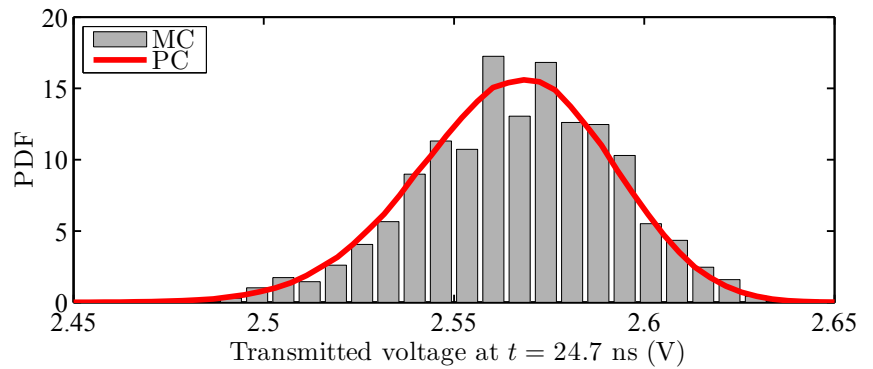

Fig. 5. Probability distribution of the trasmitted voltage at $t=24.7 \mathrm{~s}$. Gray bars: MC result; red line: PC estimation.

number of samples considered. On the contrary, thanks to the analytic polynomial expression, the PC representation allows a smoother reproduction of the PDF.

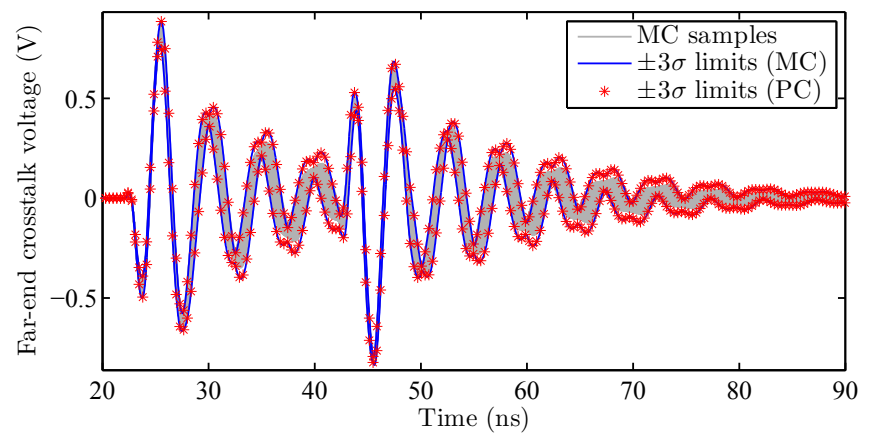

Fig. 6. Far-end crosstalk voltage in the link of Fig. 1. Curve identification is as in Fig. 4.

A similar analysis is carried out for the far-end crosstalk on the quiet line. The stochasticity of the line also causes a significant amount of variations to the voltage coupled to the adjacent line (see Fig. 6). Nonetheless, very good agreement between $\mathrm{MC}$ and $\mathrm{PC}$ in the estimation of the $\pm 3 \sigma$ limits is again established. Finally, Fig. 7 shows the PDF of the far-end crosstalk at $t=37.5$ ns. Here the PDF is very skewed compared to the Gaussian distribution of the random line parameters. Nevertheless, it is well-predicted by the PC expansion.

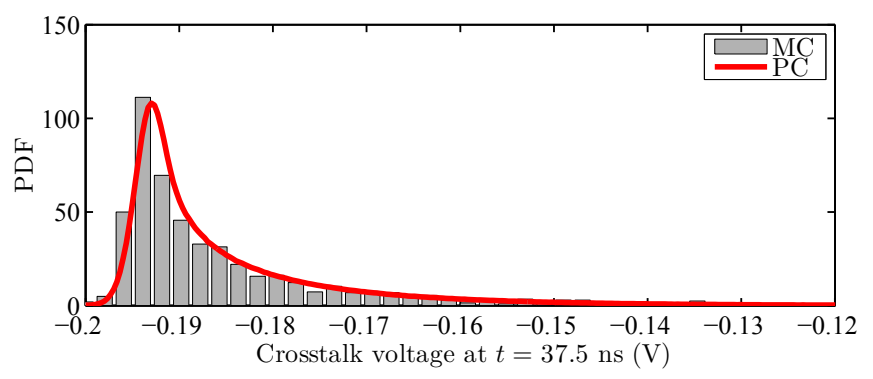

Fig. 7. Probability distribution of the far-end crosstalk voltage at $t=37.5 \mathrm{~s}$. Gray bars: MC result; red line: PC estimation.

\section{CONCLUSIONS}

This paper focuses on the statistical modeling and simulation of high-speed interconnects with uncertain physical properties and nonlinear dynamical terminations. The proposed strategy is based on the well-established polynomial chaos theory, which was proven to provide a clever solution for the stochastic analysis of lumped and distributed circuits. The unknown voltage and current variables are represented as truncated series of orthogonal polynomials of random variables. The original (stochastic) interconnect network is re-interpreted as an augmented deterministic circuit, whose simulation (e.g. by means of a standard SPICE solver) allows to efficiently compute the expansion coefficients that provide statistical features of the interconnect response.

The main achievement in this contribution is the generalization of the approach by inclusion of dynamical nonlinear terminations, e.g. representing the IC buffers. These are in fact considered as generic and complex (black-blox) multiport elements. Furthermore, any available SPICE description of the devices can be used, without modifying the proposed procedure. In the specific application, to further speed-up the simulation time, state-of-the-art behavioral macromodels are used in place of the computationally-intractable transistorlevel descriptions. A realistic coupled interconnect driven by a Flash memory chip is considered to demonstrate the proposed methodology and to stress its benefits.

\section{REFERENCES}

[1] I. S. Stievano, P. Manfredi, and F. G. Canavero, "Parameters variability effects on multiconductor interconnects via Hermite polynomial chaos," IEEE Trans. Compon. Packag. Manuf. Techol., vol. 1, no. 8, pp. 12341239, Aug. 2011.

[2] P. Manfredi, D. Vande Ginste, D. De Zutter, and F. G. Canavero, "Uncertainty assessment of lossy and dispersive lines in SPICE-type environments," IEEE Trans. Compon. Packag. Manuf. Techol., vol. 3, no. 7, pp. 1252-1258, Jul. 2013.

[3] A. Biondi, D. Vande Ginste, D. De Zutter, P. Manfredi, and F. G. Canavero, "Variability analysis of interconnects terminated by general nonlinear loads," IEEE Trans. Compon. Packag. Manuf. Techol., vol. 3, no. 7, pp. 1244-1251, Jul. 2013.

[4] P. Manfredi, D. Vande Ginste, D. De Zutter, and F. G. Canavero, "Stochastic modeling of nonlinear circuits via SPICE-compatible spectral equivalents," IEEE Trans. Circuits Syst. I, Reg. Papers (accepted for publication).

[5] A. Biondi, P. Manfredi, D. Vande Ginste, D. De Zutter, and F. G. Canavero, "Variability analysis of interconnect structures including general nonlinear elements in a SPICE-type framework," IET Electron. Lett. (accepted for publication).

[6] D. Xiu, "Fast numerical methods for stochastic computations: a review," Commun. Computational Physics, vol. 5, no. 2-4, pp. 242-272, Feb. 2009.

[7] I. S. Stievano, I. A. Maio, F. G. Canavero, and C. Siviero, "Reliable eyediagram analysis of data links via device macromodels," IEEE Trans. Adv. Packag., vol. 29, no. 1, pp. 31-38, Feb. 2006.

[8] HSPICE User Guide: Signal Integrity, Version B-2008.09, Synopsys, Inc., Mountain View, CA, USA, Sep. 2008.

[9] C. R. Paul, Analysis of Multiconductor Transmission Lines. New York: Wiley, 1994.

[10] I. S. Stievano, L. Rigazio, F. G. Canavero, T. R. Cunha, J. C. Pedro, H. M. Teixeira, A. Girardi, R. Izzi, and F. Vitale, "Behavioral modeling of IC memories from measured data," IEEE Trans. Instrum. Meas., vol. 60, no. 10, pp. 3471-3479, Oct. 2011. 